\title{
Discussing Cluster Shapes of Fuzzy Classifiers
}

\author{
Andreas Nürnberger, Aljoscha Klose, Rudolf Kruse \\ University of Magdeburg, Faculty of Computer Science \\ 39106 Magdeburg, Germany \\ E-mail: andreas.nuernberger@cs.uni-magdeburg.de
}

\begin{abstract}
Fuzzy classification rules are widely considered a wellsuited representation of classification knowledge, as they allow readable and interpretable rule bases. The goal of this paper is to discuss the shapes of the resulting classification borders and thus which class distributions can be represented by such classification systems. $2 D$ and $3 D$ visualizations are used to illustrate the cluster shapes and the borders between distinct classes. Furthermore, general hints concerning the shape of higher dimensional clusters are given.
\end{abstract}

\section{Introduction}

Fuzzy classification rules are widely considered a wellsuited representation of classification knowledge, as they allow readable and interpretable fuzzy rule bases [1]. Due to their abstraction from numbers to linguistic variables they resemble the way, humans would possibly formulate their knowledge [5]. Everybody can easily be convinced that the world is inherently fuzzy and that crisp thresholds can almost never be justified. Therefore, the use of fuzzy rules seems quite intuitive. Specifying fuzzy sets may seem easier than the specification of crisp intervals. One may hope that the inference system will 'somehow' deal with the uncertainties. Especially, when rule bases are automatically or half-automatically created from example data, this can hold true.

But finally, when the derived classifier is applied to data, usually a winner takes all principle is used to determine a single class to which the applied data is most similar. Therefore, there are always crisp borders between regions of different predicted classes.

The goal of this paper is to discuss the shape of these borders and thus which class distributions can be represented by such a system. In the following sections we investigate different influences that should be considered, when a fuzzy rule base is created (manually or automatically). These are, for example, the t-norm used, the type of fuzzy sets and the number and complexity of fuzzy rules used to define the classifier.

Our main goal is to give the potential user an intuition of what a fuzzy classifier can do and cannot do, and what should be considered when one is created. Therefore, 2D and $3 \mathrm{D}$ visualizations are used to illustrate the general shapes of borders between distinct classes and general hints concerning the shape of higher dimensional clusters are given.

First of all, we describe the general structure of fuzzy classification systems.

\subsection{Fuzzy classification systems}

The basic idea of fuzzy classification systems [1] is to describe the supposed clusters - to which the respective classes are assigned - by vague cluster prototypes. These prototypes are defined by a number of fuzzy sets which circumscribe the cluster in each considered dimension of the data space. Thus, a specific cluster $i$ is defined by a fuzzy classification rule of the form:

$$
\begin{array}{ll}
\text { if } & x_{1} \text { is } \mu_{i, 1} \text { and } x_{2} \text { is } \mu_{i, 2} \text { and } \ldots x_{n} \text { is } \mu_{i, n} \\
\text { then } & \text { pattern }\left(x_{1}, x_{2}, \ldots, x_{n}\right) \text { belongs to class } c_{k},
\end{array}
$$

where $\mu_{i, j}$ is a membership function that defines the fuzzy set describing the cluster $i$ in dimension $j$. (In the following we use for simplification the fuzzy sets $(A, B$, ...) instead of their membership functions $\mu$ to define fuzzy rules). For the evaluation of a fuzzy (classification) rule a t-norm has to be defined, by which the activation of the rule and thus the membership value of the pattern to the specific class $c_{k}$ can be derived. Common t-norms used for fuzzy classification systems are min and prod [5]. If more than one rule predicts the same class usually the highest rule activation is chosen ( $\max \mathrm{t}$-conorm).

A fuzzy classification system is defined by a rule base consisting of fuzzy classification rules. Such a system represents an approximation of an (unknown) function $\varphi: \mathbb{R}^{n} \rightarrow\{0,1\}^{m}$ that represents the classification task, where $\varphi(\mathrm{x})=\left(c_{1}, c_{2}, \ldots, c_{m}\right)$ such that $c_{i}=1$ and $c_{j}=0$ $(j \in\{1, \ldots, \mathrm{m}\}, j \neq i)$, i.e. $\mathbf{x}$ belongs to class $c_{i}$. Usually, the rule base does not approximate $\varphi$ but the function 
$\varphi^{\prime}: \mathbb{R}^{n} \rightarrow[0,1]^{m}$. We can obtain $\varphi(\mathrm{x})$ by use of an interpretation function' $\psi$, which determines one specific class: $\varphi(\mathrm{x})=\psi\left(\varphi^{\prime}(\mathrm{x})\right)$. Usually the class with the largest activation value is chosen (winner takes all principle).

\subsection{Obtaining fuzzy classifiers}

Conventional classifiers are usually derived from data and are not specified manually, due to its (mostly) complex mathematical description. In contrast to this, fuzzy classifiers make it possible to define classifiers manually and to interpret learned classifiers in an easy way. Currently, there are two common methods to learn them from data: fuzzy clustering and neuro-fuzzy learning.

Fuzzy clustering. In case of fuzzy clustering (see, for example, [1][2]) the input space is searched for clusters. The number of clusters is determined by an evaluation measure, and the size and shape of the clusters is given by the clustering algorithm. The obtained clusters can function as a fuzzy classifier, in fact, there is no need to express the classifier by rules. However, in this case the (linguistic) interpretability is lost, and therefore fuzzy rules can be created by projection of clusters [4]. This can cause a loss of information, because a fuzzy rule created by projection does not represent the cluster exactly, but only the smallest encompassing hyperbox [1]. The performance of a fuzzy classifier obtained by clustering is therefore usually reduced, once it is expressed in form of fuzzy rules.

Neuro-fuzzy learning. Another method to obtain a fuzzy classifier from data is to use neuro-fuzzy approaches (see, for example, [6][7]). These approaches create fuzzy classifiers from data by heuristic learning procedures. If the neuro-fuzzy approach meets specific restrictions, then the interpretability of the resulting classifier, and an acceptable performance might be obtained [8]. A neuro-fuzzy approach is also often less computationally expensive than a clustering approach, because of its simplicity.

In the following we have a look at the capabilities respectively the class borders of fuzzy classifiers. We focus on triangular fuzzy sets, but in many cases the results are similar if any convex fuzzy sets are used. For an analysis of arbitrary fuzzy sets see [3]. Since the interpretation of such fuzzy sets as linguistic terms is critical, it is unusual in practice and thus not considered here.

\section{Classifiers in a 2D data space}

An example of a simple fuzzy classification system consisting of the three fuzzy rules
If $x$ is $A_{1}$ and $y$ is $B_{1}$ then pattern $(x, y)$ belongs to $c_{1}$

If $x$ is $A_{2}$ and $y$ is $B_{2}$ then pattern $(x, y)$ belongs to $c_{2}$

If $x$ is $A_{3}$ and $y$ is $B_{3}$ then pattern $(x, y)$ belongs to $c_{3}$

where the fuzzy sets $A_{i}$ and $B_{i}$ are defined by triangular membership functions is depicted in Figure 1. The pyramids $R_{1}, R_{2}$ and $R_{3}$ represent the membership degrees of each data point $(x, y)$ to the respective classes $c_{i}$, obtained by use of the min t-norm. In Figure 1 (b) the resulting class borders and the contour lines of equal membership values are represented in a projection to the $(x, y)$-data space. To derive the predicted class a winner takes all principle was used.

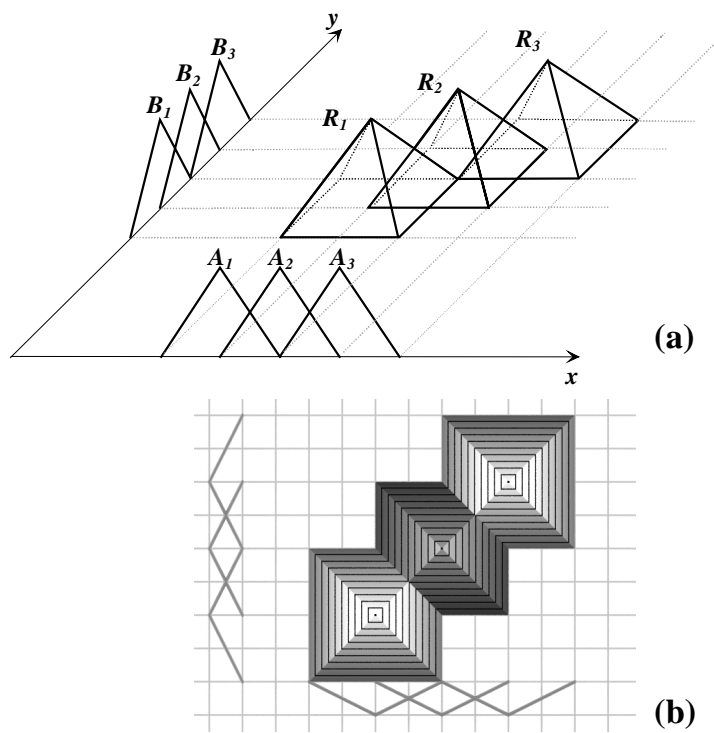

Figure 1. Representation of three fuzzy rules

\subsection{Using different t-norms}

In the example presented above the t-norm $\min$ and a winner takes all principle were used to evaluate the given rule base. In the following we present the cluster forms which could be obtained for specific rule bases using different t-norms.

Full rule base. A full rule base is often used if a neurofuzzy learning approach without pruning is applied. In this case the data space is completely covered by fuzzy rules. The classes obtained by evaluating the rule base are arranged in a regular grid - independent of the used t-norm - as depicted in Figure 3 (a) and (b). Nevertheless, the obtained membership values to the respective classes depend on the used t-norm (see contour lines in Figure 3 (a) and (b)). So, it could be useful to select an appropriate tnorm for a specific problem, even if a full rule base is used. 
The classification grid can be derived without evaluating the whole rule base by simply calculating the intersection points of two neighboring fuzzy sets in each considered dimension (assuming max t-conorm):

Let $\mathrm{T}$ be a t-norm and $A, B_{1}$, and $B_{2}$ be convex fuzzy sets and $R_{1}, R_{2}$ be the rules

$R_{1}$ : If $x$ is $A$ and $y$ is $B_{1}$ then pattern $(x, y)$ belongs to $c_{1}$

$R_{2}$ : If $x$ is $A$ and $y$ is $B_{2}$ then pattern $(x, y)$ belongs to $c_{2}$ that define two neighboring clusters, then the predicted class $c$ can be derived by

$$
c=\underset{i \in\{1,2\}}{\arg \max }\left\{\mathrm{T}\left(A(x), B_{i}(y)\right)\right\} .
$$

If $c_{1}$ is the predicted class, then $\mathrm{T}\left(A(x), B_{1}(y)\right)>$ $\mathrm{T}\left(A(x), B_{2}(y)\right)$. Thus, $B_{1}(y)>B_{2}(y)$ must apply due to the monotony of t-norms. If $c_{2}$ is the predicted class, then $B_{2}(y)>B_{1}(y)$ must apply. So the 'border' or ambiguous line between the two clusters just depends on the intersection point of $B_{1}$ and $B_{2}$. The separation line is orthogonal to the $y$-axes.

Ambiguous areas can occur if the used t-norm is not strongly monotonic. E.g. using the t-norm min can cause undefined areas (defined by patterns $(x, y)$ where $A(x)<$ $B_{1}(y)$ and $\left.A(x)<B_{2}(y)\right)$. An example using two clusters is depicted in Figure 2. Even if a full rule base is used ambiguous areas occur at the edges of every grid cell, if fuzzy partitions with different intersection levels (membership values) in the considered dimensions are used.

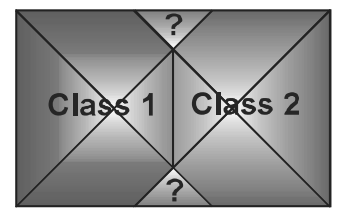

Figure 2. Ambiguous areas using min t-norm

Sparse rule base. A sparse rule base is usually obtained if neuro-fuzzy learning methods with pruning techniques or fuzzy clustering techniques were used, or specific clusters were defined manually. In this case, specific classes are described by individual but mostly overlapping areas defined by individual fuzzy rules. Hereby, the resulting borders between two classes strongly depend on the used tnorm as depicted in Figure 3 (c) and (d). The cluster shape obtained by use of the prod t-norm is much smoother than the shape obtained by use of the min t-norm. Nevertheless, in both cases the border to the 'undefined' data space is the same.

The exact class borders can be obtained by calculating the intersection line of the output of two fuzzy rules describing overlapping clusters. So, using the fuzzy rules $R_{1}$ and $R_{2}, \mathrm{~T}\left(A_{1}(x), B_{1}(y)\right)=\mathrm{T}\left(A_{2}(x), B_{2}(y)\right)$ must hold. If the t-norm $\min$ is used, then the class borders can be described - assuming no enclosing fuzzy sets - by at most three lines (see Figure 1 (b) and Figure 3 (c), (e)). If the prod tnorm is used, the borders can be described by hyperbola sections (see Figure 3 (d) and (f)).

Use of threshold membership values. If a minimal membership value is demanded to assign a data point to a specific class (reject threshold), the border to 'undefined' data space becomes different depending on the t-norm used (see the examples depicted in Figure 3 (d) and (e)). This can be seen as 'flooding' the membership data space (i.e. see the pyramids in Figure 1). In this way, even classifiers defined by full rule bases became different depending on the used t-norm if the threshold membership value is increased above the intersection level. For example, the clusters depicted in Figure 3 (a) and (b) would become 'islands', with 'coasts' according to their contour lines.

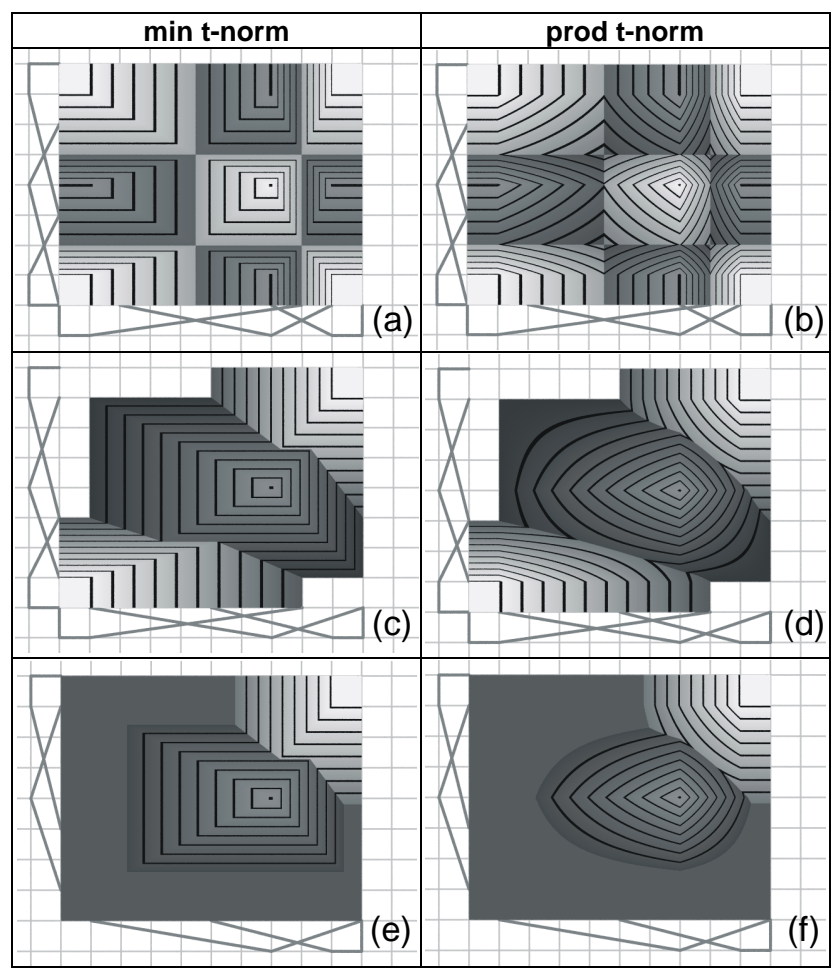

Figure 3. 2D cluster shapes

\section{Classifiers in $3 D$ data space}

To obtain usable diagrams of three dimensional fuzzy clusters we omit the (direct) representation of the membership degrees. The clusters were shown by illuminated plots of the outer cluster surfaces in the 3D data space. To improve the evidence of the description, the plots are rotated. Nevertheless, all single clusters created by fuzzy classifiers are spanned by the fuzzy sets in the antecedents of the rule. Therefore, the axes of the spanning system are always parallel to the axes of the data space. 
Furthermore, for most of the commonly used t-norms holds: $a>0 \wedge b>0 \Rightarrow T(a, b)>0$. So, the outer shape of the cluster is usually defined by an axes parallel hyper box (see Figure 4 (a)).

The presented plots in Figure 4 (a) to (j) were obtained by use of two fuzzy rules:

$$
\begin{aligned}
& \text { If } x \text { is } A_{1} \text { and } y \text { is } B_{1} \text { and } z \text { is } C_{1} \\
& \text { then pattern }(x, y, z) \text { belongs to } c_{1} \\
& \text { If } x \text { is } A_{2} \text { and } y \text { is } B_{2} \text { and } z \text { is } C_{2} \\
& \text { then pattern }(x, y, z) \text { belongs to } c_{2}
\end{aligned}
$$

where $A_{i}, B_{i}$ and $C_{i}$ are fuzzy sets. In Figure $4(\mathrm{k})$ and (l) just one rule was used.

Full rule base. Using a full rule base the resulting classes obtained by evaluation of the rule base are arranged in a regular grid, independent of the used t-norm, as in the two dimensional case.

Sparse rule base. If a sparse rule base is used, the obtained cluster forms depend on the used t-norm, if two clusters overlap or a threshold value is used - as in the two dimensional case. Figure 4 (a) represents the outer shape of two overlapping clusters, which are obtained by the use of triangular symmetric fuzzy sets in each dimension and no threshold value. If a threshold value is used the total shape depends on the used t-norm, as depicted in (b). If the threshold value is increased, the overlapping of the clusters can be reduced or even canceled as depicted in (c) and (d). Additionally, these figures show the membership degrees on a (orthogonal) plane in data space. The darker areas describe a small membership degree, the lighter areas a high membership degree to the respective clusters. As expected, the distribution of the membership values is similar to the two dimensional case (see Figure 3).

If overlapping clusters define different classes, the obtained class borders depend on the used t-norm: plane sections, if $\min$ (see (e)) or hyperboloid sections if prod is used (see (f)).

Examples of the influence of t-conorms are depicted in (g) to $(\mathrm{j})$. The bounded sum t-conorm $\min \left(1, \Sigma \mu_{i}\right)$ was used for two rules predicting the same class. In this case, the clusters which are defined by overlapping fuzzy sets are connected by a 'tunnel' even if a threshold value would separate the individual clusters (compare to (c) and (d)). As shown in (h) the use of the bounded sum can lead to high membership values in the overlapping areas, especially if the fuzzy sets do not overlap symmetrically at membership value 0.5 , as in this example.

To obtain elongated clusters trapezoidal fuzzy sets can be used. In (k) and (l) clusters defined by two triangular and one trapezoidal fuzzy set are shown. Nevertheless, the obtained clusters are parallel to the axes of the data space.

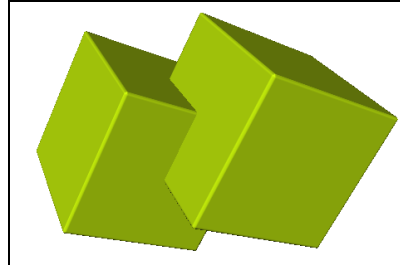

(a) surface level $=0$, any t-norm with $a>0 \wedge b>0 \Rightarrow T(a, b)>0$

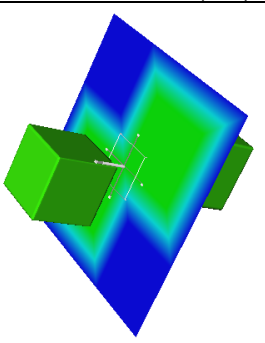

(c) min t-norm, surface level $=0.55$

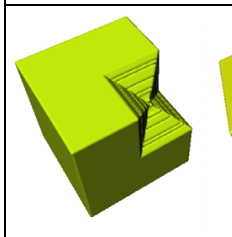

(e) $\min$ t-norm, surface level=0, symmetrical and unsymmetrical overlapping

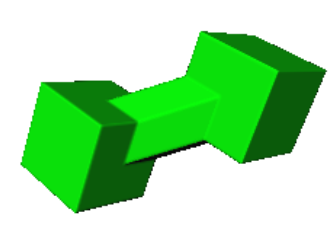

(g) sym. overl., min t-norm, surface level $=0.5$,

t-conorm for class: $\min \left(1, \mu_{a}+\mu_{b}\right)$ t-conorm for class: $\min \left(1, \mu_{a}+\mu_{b}\right)$

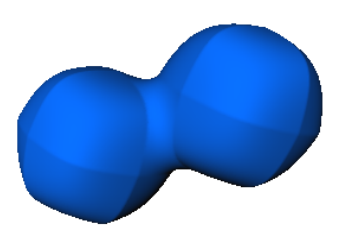

(i) sym. overl., prod t-norm, surface level $=0.1$, $\mathrm{t}$-conorm for class: $\min \left(1, \mu_{\mathrm{a}}+\mu_{\mathrm{b}}\right) \mathrm{t}$-conorm for class: $\min \left(1, \mu_{\mathrm{a}}+\mu_{b}\right)$

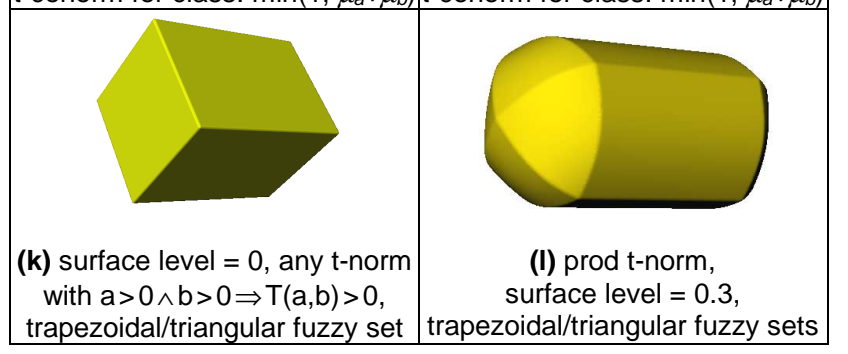

Figure 4. 3D Cluster Shapes

(b) prod t-norm, surface level $=0.05$

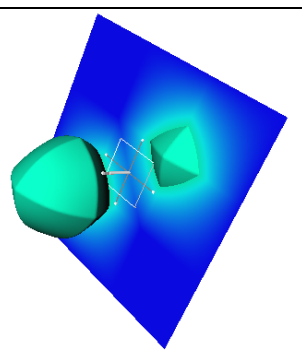

(d) prod t-norm, surface level $=0.3$

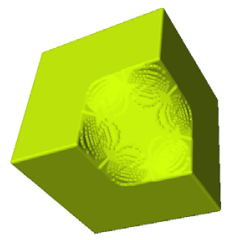

(f) prod t-norm, surface level $=0.0$

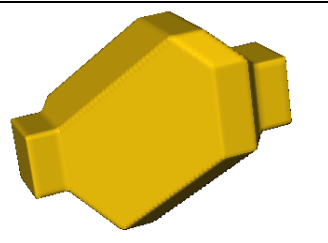

(h) unsym. overl., min t-norm, surface level $=0.8$,

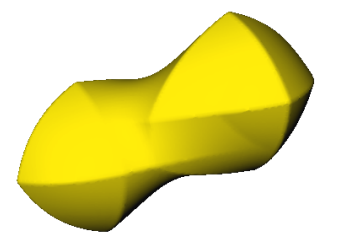

(j) unsym. overl., prod t-norm, surface level $=0.4$

(I) prod t-norm

with $a>0 \wedge b>0 \Rightarrow T(a, b)>0$ trapezoidal/triangular fuzzy set trapezoidal/triangular fuzzy sets 


\subsection{Higher dimensional data space}

Using a full rule base in n-dimensional data space results in a regular $\mathrm{n}$-dimensional grid. If a sparse rule base is used, cluster forms and borders can be derived as presented above. Unfortunately, in higher dimensional data space the prod t-norm results in a very low rule activation at the cluster borders, since the activation of a rule $R$ depends exponentially from the number of antecedents:

$$
\operatorname{prod}(R)=\prod_{i=1, \ldots, n} A_{i}\left(x_{i}\right)
$$

An example showing the membership degrees based on the distance to the center of a cluster defined by equal triangular fuzzy sets in each considered dimension is presented in Figure 5.

If a rule base with fuzzy rules consisting of different numbers of antecedents is used - which usually occurs if pruning techniques were applied - the prod t-norm can lead to different cluster sizes even if the same linguistic terms respectively fuzzy sets were used (i.e. in overlapping areas). Especially, if a threshold value is used it will be nearly impossible to define such classifiers manually. Even the interpretability of learned classifiers usually will be poor.

These effects do not occur if the t-norm $\min$ is used. So, the advantages and disadvantages of both t-norms should be carefully considered if a fuzzy classification system is created.

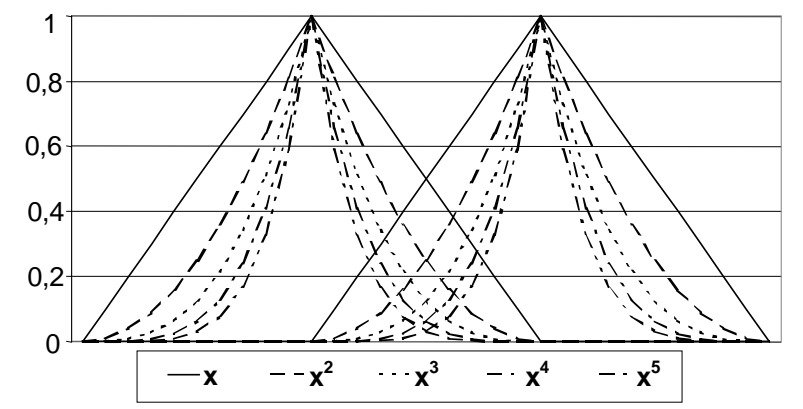

Figure 5. Rule activation in higher dimensional data space using the t-norm prod

\section{Conclusion}

This paper tried to give an insight into the impacts of factors like the density of rules, the used t-norm and tconorm, the number of antecedents or reject thresholds on the representation ability of fuzzy classifiers.

One remarkable result is that fully covered rule bases or fully covered parts of rule bases - can only represent axes parallel class borders, mostly due to the restriction to the fuzzy sets of the partitioning. Thus, less rules give more flexibility and may enable the classifier to represent the actual class distribution of the data.
If a rejecting classifier is needed, the definition of a minimal membership threshold can be useful. As shown in Section 2 and 3 the use of e.g. prod as t-norm and/or bounded sum as t-conorm leads to cluster forms that seem to be more natural or intuitive in comparison to the axes parallel hyperboxes that result from the commonly used min-max-inference.

The borders between classes are - as well as the borders to the reject class - smoother for t-norm prod in comparison to the piecewise linear borders resulting from $\mathrm{min}$.

However, mainly a classifier is not intended to 'look' nice, but to separate the classes correctly. In most cases this can as well be done with min-max-inference. The $t-$ norm min has the considerable advantage that it behaves rather insensible to the number of dimensions, as it is the only absorbing t-norm. This allows pruning techniques that reduce the number of antecedents in the individual rules. Other t-norms cause the average activation to decrease rapidly with a growing number of antecedents (see Figure 5).

In general, fuzzy classifiers are not meant to outperform other classification approaches. This is mainly prevented by the usually small numbers of linguistic terms that are shared by all rules. Nevertheless, this voluntary restriction is substantial for the interpretability and readability of the rule base. This is widely considered more important than the 'last percent' of classification performance.

\section{References}

[1] F. Höppner, F. Klawonn, and R. Kruse. Fuzzy Clusteranalysis. John Wiley \& Sons Ltd., Chichester, 1999.

[2] D. Gustafson, and W. Kessel. Fuzzy Clustering with a Fuzzy Covariance Matrix. In Proc. IEEE CDC, 761-766, San Diego, CA, 1979.

[3] F. Klawonn, and E.-P. Klement. Mathematical Analysis of Fuzzy Classifiers. In: X. Liu, P. Cohen, M. Berthold (eds.): Advances in Intelligent Data Analysis. Springer, Berlin, 359-370, 1997.

[4] F. Klawonn, and R. Kruse. Constructing a Fuzzy Controller from Data. Fuzzy Sets and Systems, 85:177-193, 1997.

[5] R. Kruse, J. Gebhardt, F. Klawonn. Foundations of Fuzzy Systems, John Wiley \& Sons, Inc., New York, Chichester, et al., 1994.

[6] C.T. Lin, and C.S.G. Lee. Neural Fuzzy Systems: A NeuroFuzzy Synergism to Intelligent Systems. Prentice-Hall Inc., New Jersey, London, et. al., 1996.

[7] D. Nauck, F. Klawonn, and R. Kruse. Foundations of Neuro-Fuzzy Systems. John Wiley \& Sons Inc., New York, 1997.

[8] D. Nauck, A. Nürnberger, and R. Kruse: Neuro-Fuzzy Classification. In: Advances in Data Science and Classification, Proc. 6th Conf. of the Int. Federation Classification Societies (IFCS-98), University La Sapienza, Juli 21-24, Rom, Italy, Springer, Berlin, 1998. 